\title{
Viscous effects in time-dependent planar reconnection
}

\author{
C. K. Armstrong, I. J. D. Craig, and Y. E. Litvinenko
}

\author{
Department of Mathematics, University of Waikato, P. B. 3105, Hamilton, New Zealand \\ e-mail: [cka3;i.craig;yuril]@waikato.ac.nz
}

Received 8 July 2011 / Accepted 23 August 2011

\begin{abstract}
Context. Viscous dissipation is expected to play a significant part in energy release in solar flares, yet the role of viscosity in a weakly resistive plasma of the solar corona remains unclear.

Aims. We attempt to clarify the role of viscous effects in magnetic reconnection by performing simulations of reconnection in planar periodic geometry in an incompressible viscous resistive plasma.

Methods. We consider magnetic reconnection, driven by large-scale vortical flows. We use both the classical shear viscosity and the Braginskii form for the ion parallel viscosity in a magnetised plasma. We determine the scalings of the current sheet parameters and the global rates of resistive and viscous dissipation. We use steady-state exact solutions and scaling arguments to interpret the numerical results.

Results. We show that, regardless of the form of viscosity, the resistive non-viscous analytical solutions for flux pile-up merging provide a very good approximation of the numerical results in the reconnecting current sheet. We find no evidence for a viscoresistive scale. Numerical results for a highly sheared magnetic field, however, appear to deviate from the analytical predictions in the case of the Braginskii viscosity.
\end{abstract}

Key words. magnetic reconnection - magnetohydrodynamics (MHD) - Sun: flares

\section{Introduction}

Viscous effects are expected to play a significant part in a wide variety of dynamic phenomena in the solar convection zone (e.g, Rüdiger \& Kitchatinov 1997), in the solar atmosphere (e.g., Hollweg 1985), and in the solar wind (Montgomery 1992). Of particular interest is the role of viscosity in the process of magnetic reconnection in the solar corona (Hollweg 1986). The classical electric resistivity $\eta$ of the high-temperature coronal plasma is so low that additional non-ideal processes are needed, for instance, to explain the observed rapid energy release in solar flares (for a review, see Priest \& Forbes 2000).

Despite a number of studies, the role of viscosity in magnetic reconnection remains unclear. On the one hand, SweetParker (Parker 1957) style arguments suggest that a sufficiently large classical shear viscosity $v$ leads to thicker reconnecting current sheets, slower reconnection inflows, and slower reconnection outflows (Park et al. 1983). Notably, the scaling $\sim(\eta v)^{1 / 4}$ is predicted for the thickness of the current sheet, which should be contrasted with the usual resistive Sweet-Parker scaling $\sim \eta^{1 / 2}$. The visco-resistive scaling also emerges in an analytical model for slow magnetic reconnection at a two-dimensional neutral point (Titov \& Priest 1997). On the other hand, a number of solutions for steady magnetic merging in viscous resistive plasmas neither contain a visco-resistive scale nor predict the slowdown of the reconnection flow due to viscosity (e.g., Sonnerup \& Priest 1975; Besser et al. 1990; Jardine et al. 1992).

The picture is further complicated by the fact that the classical isotropic viscosity, while adequately representing viscous effects in a weakly magnetised plasma (e.g., Rüdiger \& Kitchatinov 1997), is inaccurate in a strongly magnetised plasma such as the solar corona. For a coronal plasma in which the mean time between momentum-changing collisions exceeds the inverse proton gyrofrequency, the Braginskii (1965) viscosity that accounts for the anisotropies introduced by the magnetic field is more accurate (Hollweg 1986). Exact analytical solutions and scaling arguments, based on the Braginskii (1965) viscosity, predict that the current sheet parameters are independent of the viscosity coefficient (Litvinenko 2005; Craig \& Litvinenko 2009, 2010).

The contradictory results of the steady analytical models motivate us to investigate the structure of the reconnection region in an incompressible visco-resistive plasma, using numerical simulations of time-dependent magnetic reconnection. Numerical results and scaling arguments suggest the effect of compressibility is relatively minor for the flow driven merging solutions discussed here (Litvinenko \& Craig 2003). Accordingly, we investigate reconnection in a "closed" twodimensional doubly periodic reconnection region, thus simulating advection-driven reconnection. We also use scaling arguments to interpret our numerical results.

The paper is organised as follows. We introduce the incompressible magnetohydrodynamic (MHD) equations and discuss resistive and viscous dissipation processes in Sect. 2. We summarise the available analytical solutions and scaling law arguments for steady magnetic merging in Sect. 3. In Sect. 4, we describe our simulations of time-dependent magnetic reconnection. We use the numerical results to obtain scalings for both the local current sheet parameters and the global rates of resistive and viscous dissipation. We specifically compare the effects of the classical viscosity and the Braginskii viscosity. We also present an exploratory analysis of the case of strongly sheared reconnecting fields. In Sect. 5 we present our conclusions. 


\section{Formulation of the problem}

\subsection{MHD equations}

The MHD system for an incompressible resistive viscous plasma consists of the momentum and induction equations, supplemented by the divergence-free constraints for the velocity $\boldsymbol{v}$ and magnetic field $\boldsymbol{B}$. In dimensionless form we have

$$
\begin{aligned}
\frac{\partial \boldsymbol{v}}{\partial t}+(\boldsymbol{v} \cdot \nabla) \boldsymbol{v} & =(\nabla \times \boldsymbol{B}) \times \boldsymbol{B}-\nabla p+\nabla \cdot \mathcal{S}, \\
\frac{\partial \boldsymbol{B}}{\partial t} & =\nabla \times(\boldsymbol{v} \times \boldsymbol{B})+\eta \nabla^{2} \boldsymbol{B}, \\
\nabla \cdot \boldsymbol{v} & =\nabla \cdot \boldsymbol{B}=0 .
\end{aligned}
$$

Here $p$ is the plasma pressure and $\eta$ the normalised resistivity. The viscous force is defined by the stress tensor $\mathcal{S}$ whose form is discussed below. The MHD equations have been scaled using the typical coronal parameters: magnetic field strength $B_{\mathrm{c}}=10^{2} \mathrm{G}$, length scale $l_{\mathrm{c}}=10^{9.5} \mathrm{~cm}$, and number density $n_{\mathrm{c}}=10^{9} \mathrm{~cm}^{-3}$. Velocities are expressed in units of the Alfvén speed $v_{\mathrm{A}}=B_{\mathrm{c}} /\left(4 \pi \rho_{\mathrm{c}}\right)^{1 / 2} \simeq 10^{9} \mathrm{~cm} \mathrm{~s}^{-1}$, where $\rho_{\mathrm{c}}=m_{\mathrm{p}} n_{\mathrm{c}}$ is the mass density and $m_{\mathrm{p}}$ is the proton mass.

Below we consider two alternative forms for the viscosity tensor: the classical isotropic viscosity

$\mathcal{S}_{i j}=v\left(\partial_{j} v_{i}+\partial_{i} v_{j}\right)$

and the Braginskii (1965) form for the ion parallel viscosity

$\mathcal{S}_{i j}=v\left(3 \frac{B_{i} B_{j}}{B^{2}}-\delta_{i j}\right)\left(\frac{B_{k} B_{l}}{B^{2}} \partial_{l} v_{k}\right)$.

As usual, summation over repeated indices is assumed. Although the form (4) is commonly employed, it becomes inaccurate in a magnetised plasma such as the solar corona. In such cases the Braginskii form that accounts for the anisotropies introduced by the magnetic field is more accurate (Hollweg 1986).

Two dimensionless parameters control the rate of energy dissipation, the resistivity $\eta$ and the viscosity $v$. Notably, although both are $\ll 1$, the coronal temperature interval $(2-10) \times 10^{6} \mathrm{~K}$ implies that $v \gg \eta$, and so viscous dissipation is likely to dominate resistive dissipation in solar flares (e.g., Craig \& Litvinenko 2009 , and references therein). We use $W_{\mathrm{c}}=v_{\mathrm{A}} l_{\mathrm{c}}^{2} B_{\mathrm{c}}^{2} /(8 \pi) \simeq$ $4 \times 10^{30} \mathrm{erg} \mathrm{s}^{-1}$ to scale the global energy dissipation rate. The dimensionless rate of resistive (Ohmic) dissipation is given by

$W_{\eta}=\int \eta J^{2} \mathrm{~d} V$

where $\boldsymbol{J}=\nabla \times \boldsymbol{B}$ is the current density. The viscous dissipation rate is given by

$W_{v}=\int S_{i j} \partial_{j} v_{i} \mathrm{~d} V$

\subsection{Planar momentum and induction equations}

Planar solutions for the magnetic and velocity fields can be expressed using flux and stream functions:

$\boldsymbol{B}=\nabla \psi(x, y, t) \times \hat{z}, \quad \boldsymbol{v}=\nabla \phi(x, y, t) \times \hat{z}$.

Since these forms satisfy Eq. (3) identically, the MHD equations reduce to

$$
\begin{aligned}
\frac{\partial}{\partial t}\left(\nabla^{2} \phi\right)+\left[\nabla^{2} \phi, \phi\right] & =\left[\nabla^{2} \psi, \psi\right]+G, \\
\frac{\partial \psi}{\partial t}+[\psi, \phi] & =\eta \nabla^{2} \psi,
\end{aligned}
$$

where we use the Poisson bracket notation typified by

$[\psi, \phi]=\partial_{x} \psi \partial_{y} \phi-\partial_{y} \psi \partial_{x} \phi$

Viscous effects are represented by

$G=-(\nabla \times \nabla \cdot \mathcal{S}) \cdot \hat{z}$.

\section{Analytical models of magnetic merging}

\subsection{An exact visco-resistive model: classical viscosity}

The simplest exact solution for magnetic merging is that of Sonnerup \& Priest (1975). A stagnation point flow supports the steady annihilation of straight field lines advected towards a strong current layer centred on $x=0$. Specifically the MHD equations are satisfied by

$$
\begin{aligned}
\boldsymbol{v} & =-\alpha x \hat{\boldsymbol{x}}+\alpha y \hat{\boldsymbol{y}}, \\
\boldsymbol{B} & =\frac{E}{\eta} \exp \left(-\frac{\alpha x^{2}}{2 \eta}\right) \int_{0}^{x} \exp \left(\frac{\alpha s^{2}}{2 \eta}\right) \mathrm{d} s \hat{\boldsymbol{y}} \\
& =\frac{E}{\eta \mu} \operatorname{daw}(\mu x) \hat{\boldsymbol{y}},
\end{aligned}
$$

where the Dawson function is defined by

$\operatorname{daw}(s)=\int_{0}^{s} \exp \left(u^{2}-s^{2}\right) \mathrm{d} u$

and $\mu^{2}=\alpha / 2 \eta$. Here $\alpha$ determines the amplitude of the velocity field and $E$ is a uniform electric field in the z-direction. The Dawson function identifies

$x_{s} \simeq \sqrt{\frac{\eta}{\alpha}}$

as the thickness of the current layer. This result implies the inflow and outflow speeds associated with the current sheet $v_{\text {in }} \simeq \sqrt{\alpha \eta}$ and $v_{\text {out }} \simeq \alpha$. This solution can be generalised to include vortical and shear flows (Besser et al. 1990; Jardine et al. 1992). Another generalisation, valid only if $v=0$, is to allow the merging of curved field lines, leading to $X$-point reconnection (Craig \& Henton 1995). None of these refinements significantly changes the scaling laws derived below.

Using Eqs. (4) and (7), the classical viscous dissipation rate in volume $V$ is given by

$W_{v}=4 v \alpha^{2} V$.

(In our numerical simulations, the volume is that of the cube $0 \leq|x|,|y|,|z| \leq 1$.) The resistive dissipation is easy to estimate if the thinness of the sheet is exploited. Thus if $B_{s}$ is the peak field strength in the current layer, then $J \simeq B_{s} / x_{s}$ is the current density, and we have from Eq. (6) that

$W_{\eta} \simeq(\alpha \eta)^{1 / 2} B_{s}^{2} V$.

Similarly, the rate of flux annihilation is

$E=\eta J \simeq(\alpha \eta)^{1 / 2} B_{s}$.

The key aspect of these results is the presence of a single small scale $x_{s}$ determined by the resistivity. Although viscous losses do occur, these arise from maintaining the global velocity field that drives the merging. By comparing Eqs. (15) and (16) and treating $\alpha$ and $B_{s}$ as parameters of order unity, we see that viscous dissipation should dominate when $v>\eta^{1 / 2}$. More physically, if 
we assume (as in Sweet-Parker merging) that exhaust speeds are related to the strength of the current layer, then it follows that $\alpha \simeq B_{s}$ (see Litvinenko \& Craig 2000, for details), and so

$$
\frac{W_{v}}{W_{\eta}} \simeq \frac{4}{B_{s}^{1 / 2}} \frac{v}{\eta^{1 / 2}}
$$

A similar result is obtained when the Braginskii viscosity is considered, as we now demonstrate.

\subsection{An exact visco-resistive model: Braginskii viscosity}

Exact solutions for magnetic merging, supported by vortical flows, have been obtained for the case of Braginskii viscosity. Both planar merging (Litvinenko 2005) and merging driven by three-dimensional flows (Craig \& Litvinenko 2009) have been analysed.

We consider only the simplest planar solution, namely

$$
\begin{aligned}
\boldsymbol{v} & =-\frac{\alpha \sin (\omega x)}{\sin (\omega)} \hat{\boldsymbol{x}}+\frac{\alpha \omega y \cos (\omega x)}{\sin (\omega)} \hat{\boldsymbol{y}} \\
\boldsymbol{B} & =\frac{E}{\eta} \exp \left(\frac{\alpha \cos (\omega x)}{\eta \omega \sin (\omega)}\right) \int_{0}^{x} \exp \left(-\frac{\alpha \cos (\omega s)}{\eta \omega \sin (\omega)}\right) \mathrm{d} s \hat{\boldsymbol{y}} .
\end{aligned}
$$

Here the parameter $0 \leq \omega<\pi$ determines the velocity gradient on the inflow boundary. Note that, as $\omega \rightarrow 0$, the stagnationpoint flow of the previous section is recovered. The parameters of the current layer (namely its thickness $x_{s}$ and the inflow $v_{\text {in }}$ and outflow $v_{\text {out }}$ speeds) are all found to scale as those in the merging solution based on the classical viscosity.

The dimensionless dissipation rate, associated with the Braginskii viscosity, follows from Eqs. (5) and (7):

$W_{v}=3 v \alpha^{2}\left(\frac{2 \omega^{2}+\omega \sin 2 \omega}{4 \sin ^{2} \omega}\right) V$,

which reduces to $3 v \alpha^{2} V$ as $\omega \rightarrow 0$. Since the reconnection rate and Ohmic dissipation rate are essentially unchanged from the previous model (at least provided $\omega$ does not approach $\pi$ ), we again recover Eq. (18) within factors of order unity.

\subsection{The visco-resistive current layer}

The previous two cases are based on exact solutions for largescale velocity fields that drive the merging. By way of contrast, the following argument depends mainly on the local properties of the current layer. In particular, as in Park et al. (1983) and Craig \& Litvinenko (2010), Sweet-Parker style arguments can be used to infer the emergence of a visco-resistive scale in the case $v \gg \eta$.

The argument is based on mass continuity, $v_{\text {in }}=x_{s} v_{\text {out }}$, steady flux transfer, $v_{\text {in }} B_{s}=\eta B_{s} / x_{s}$, and pressure balance along and across the sheet. For a sufficiently large $v$, the classical shear viscosity slows down the outflow and thickens up the current layer with the result that

$x_{s} \simeq(\eta v)^{1 / 4} B_{s}^{-1 / 2}$.

The scaling for viscous dissipation is now based on the assumption that viscous damping is effective only within the narrow current layer-what happens outside the sheet is effectively neglected. The resulting dissipation scalings

$\eta J \simeq \eta^{3 / 4} v^{-1 / 4} B_{s}^{3 / 2}, \quad W_{\eta} \simeq W_{v} \simeq \eta^{3 / 4} v^{-1 / 4} B_{s}^{5 / 2}$, are quite different from the previous exact analytical models. These scalings (which correct a misprint in Eq. (20) of Craig \& Litvinenko 2010) suggest that a large classical viscosity $v \gg \eta$ should slow down the rate of magnetic merging. Note that when $v=\eta$ we obtain the traditional resistive Sweet-Parker scalings.

It is interesting that a similar analysis based on the Braginskii viscosity does not lead to the emergence of a visco-resistive scale, as pointed out by Craig \& Litvinenko (2010). However, the scale $x_{s} \sim(\eta v)^{1 / 4}$ has also been obtained in an analytical model of slow magnetic reconnection at a two-dimensional $X$-point (Titov \& Priest 1997), as well as in numerical studies of $X$-point collapse for both classical and Braginskii viscosities (Craig et al. 2005; Craig 2010).

To summarise, exact reconnection solutions exist for both classical and Braginskii viscosities, which do not contain a visco-resistive scale. By contrast, some analytical models and $X$-point simulations predict the emergence of the visco-resistive scale $x_{s} \sim(\eta v)^{1 / 4}$. Moreover, Sweet-Parker style scaling arguments imply that this scale should almost universally emerge in a viscous plasma with $v>\eta$.

\section{Visco-resistive simulations of magnetic reconnection}

\subsection{Numerical simulation}

In this section we describe a series of side-by-side, flow-driven reconnection experiments using the Braginskii and classical viscosities. Typically the viscous coefficient $v$ is fixed at some representative value and scalings are derived by systematically reducing the resistivity $\eta \ll v$. To provide a further check on the results we also perform a "control" resistive simulation, based on the common numerical expedient of setting $v=\eta$. That is, we would like to compare the visco-resistive reconnection scalings with those in the case $v=\eta$, but, as is well known, setting $v=0$ is susceptible to numerical difficulties. Accordingly we set $v=\eta$ to approximate the purely resistive case (Biskamp 1994; Craig \& Litvinenko 2010). Our aim is two-fold: first, to explore the emergence, or otherwise, of a visco-resistive scale; second, to quantify the differences that develop when classical and Braginskii viscosities are employed.

The results are obtained by numerically solving Eqs. (9) and (10), using a doubly periodic code over the region $0 \leq|x|$, $|y| \leq 1$. Initial conditions are derived by assuming a vortical flow velocity field (clockwise in the first quadrant) given by

$\phi(x, y, 0)=-\frac{\alpha_{0}}{\pi} \sin (\pi x) \sin (\pi y)$

where $\alpha_{0}>0$ sets the amplitude of the initial flow. We assume an initial magnetic disturbance of the form

$\psi(x, y, 0)=\frac{\beta_{0}}{\pi} \sin (\pi x) \sin (\pi y)+\frac{g_{0}}{\pi} \cos (\pi x)$,

where $g_{0}>0$ sets the amplitude of the magnetic disturbance and $\beta_{0} \geq 0$ controls the level of magnetic shear in the merging. In the simplest case of "head on" reconnection $\left(\beta_{0}=0\right)$, initially straight field lines, washed together by the inflow, rapidly evolve, forming a well defined current layer centered on the origin.

To obtain resistive scalings, the parameters $\alpha_{0}, \beta_{0}$ and $v$ are held fixed while $\eta$ and $g_{0}$ are systematically reduced. Note that simply reducing $\eta$ while keeping $g_{0}$ fixed would lead to high pressure, flux pile-up current sheets that would feed back on the flow and stall it. Accordingly, $g_{0}$ is adjusted to ensure that peak fields $B_{s}$ in the reconnecting current layers have magnitudes of 


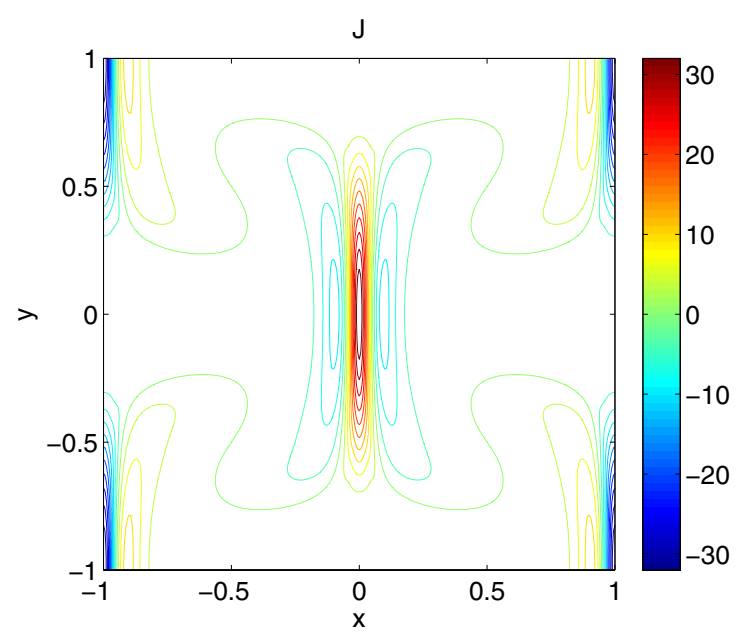

Fig. 1. Contour plot of $J$ taken at the time of maximum current density at the origin. The parameters are $\alpha_{0}=1, \beta_{0}=0$ and $\eta=v=0.004$.

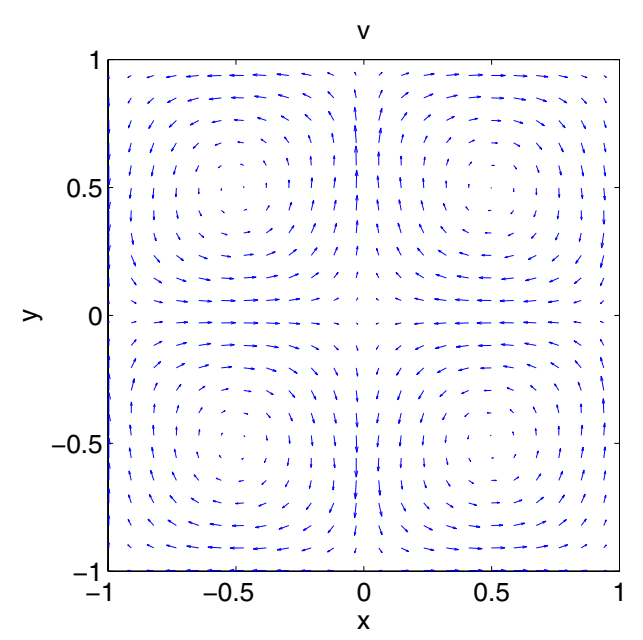

Fig. 2. Arrow plot of $v$ taken at the time of maximum current density at the origin. The parameters are the same as in Fig. 1, namely $\alpha_{0}=1$, $\beta_{0}=0$ and $\eta=v=0.004$. For visual clarity only selected velocity vectors are displayed.

order unity. This allows us to obtain scalings at resistivities that are limited only by numerical resolution in the computation (the lowest values of resistivity considered here require mesh sizes $\left.\$ 10^{-3}\right)$. In practice we take $\alpha_{0}=1$ and $v=0.004$ and allow resistivities in the range $10^{-4.5} \leq \eta \leq 10^{-2}$. Most of our simulations apply to head-on reconnection, $\beta_{0}=0$, but some preliminary results for sheared reconnection are given in Sect. 4.4.

As a final point we note that the Braginskii viscosity tensor (5) cannot be applied to fields that are very weak. Since our computation involves null points, it is convenient to adopt a form that effectively interpolates between the classical and Braginskii viscosities. In practice we adopt the "Liley" form of Hosking \& Marinoff (1973).

\subsection{The reconnecting current sheet}

Figures 1 and 2 show an example of a head-on Braginskii viscosity simulation with parameters $\alpha_{0}=1, \beta_{0}=0$ and $\eta=v=$ 0.004 . The dominant feature, which reaches maximum development after approximately one Alfvén time in the present simulation, is a strong current sheet centred on the origin. As discussed

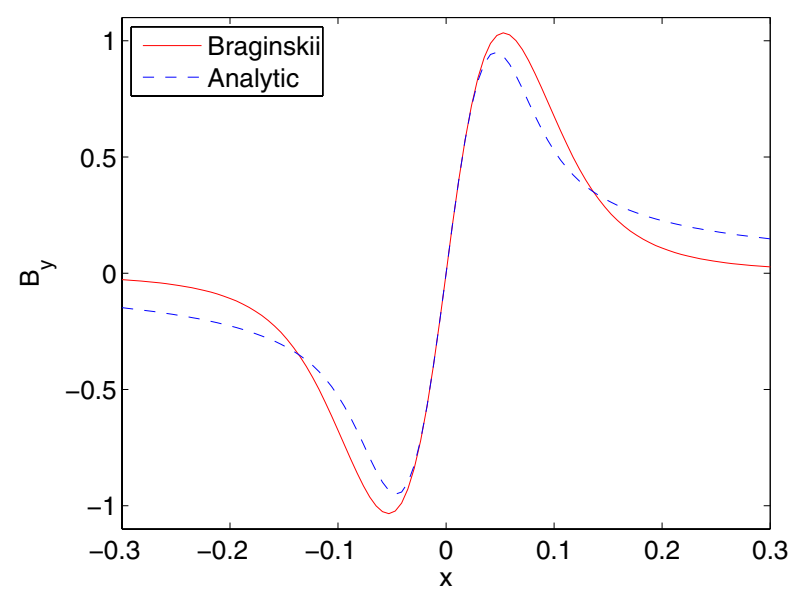

Fig. 3. The $y$-component of the magnetic field in the current layer in the case of head-on merging. The parameters are the same as in Figs. 1 and 2 . The solid line is the numerical magnetic field and the dashed line is the analytical solution from (13).

by Heerikhuisen et al. (2000), there is a good deal of numerical evidence to suggest that the properties of the current layer, when taken at the time of maximum current, can be accurately described by the steady merging models such as those discussed in Sect. 3.

More specifically, suppose we use the fields displayed in Figs. 1 and 2 to evaluate the rate of flux transfer $E$ and the inflow velocity amplitude $\alpha_{0}$. The analytical solution (13) based on these parameters can then be compared with the simulated current layer. Figure 3 displays a slice of the simulated current sheet alongside the analytical model. Despite the fact that the analytic model is purely resistive and the vorticity parameter $\omega=0$, we see that the model provides a good representation of the dynamically evolved field in the current layer. The most obvious variations occur in the outer regions dominated by the large-scale vortical flows-the regions not accurately represented by the analytic, linear inflow model of (13). The implication is that Braginskii viscosity simulations are unlikely to undermine the form of the purely resistive current layer.

\subsection{Head-on magnetic merging simulations}

We now turn to quantifying the key parameters $v_{\text {out }}, v_{\text {in }}$, and $x_{s}$ of the current layer and relating these to the global Ohmic and viscous energy release rates. Current sheet thickness is measured along the $x$ axis at the level of half current maximum. Inflow and outflow velocities are measured on the $x$ and $y$ axes respectively at the current half maximum level. Figure 4 shows a comparison of the classical, Braginskii and $v=\eta$ control current sheet outflow speeds, calculated at the time of maximum current. Minor variations are apparent, and the outflow is generally slower in the case of the classical viscosity, yet the outflow speeds are essentially invariant with $\eta$. In particular, there is no evidence for a slowdown, $v_{\text {out }} \sim(\eta / v)^{1 / 2}$, which would be caused by a viscoresistive scale in the case of classical viscosity (Sect. 3.3). The analytical scalings of Sect. 3.1 are clearly more accurate

This interpretation is borne out by an analysis of the inflow speeds $v_{\text {in }}$ and the current sheet thickness $x_{s}$, which both scale as $\eta^{1 / 2}$, as confirmed by the plots in Figs. 5 and 6 . In both cases it is very difficult to distinguish the Braginskii, classical and $v=$ $\eta$ control plots. The classical shear viscosity does provide the 


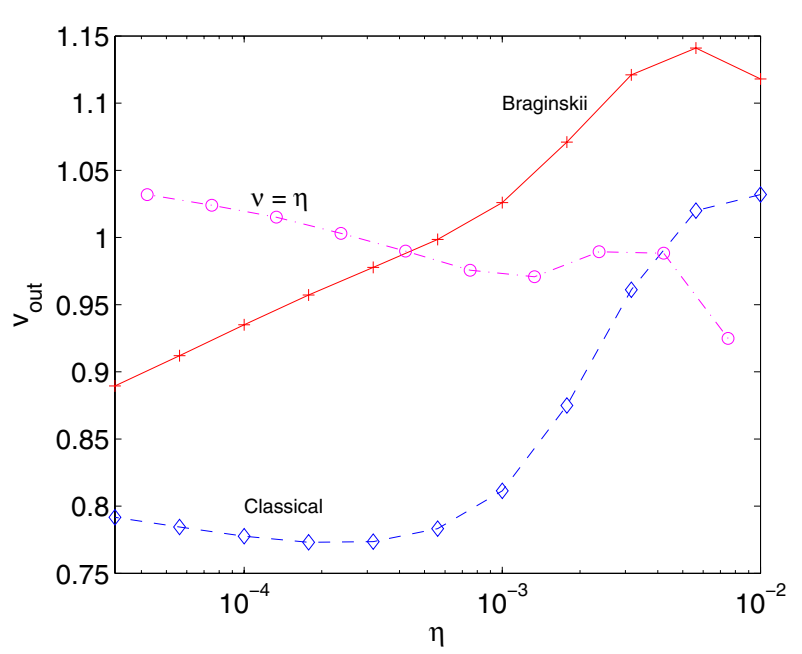

Fig. 4. Current sheet outflow speed comparisons. Crosses, diamonds and circles refer to Braginskii, classical and $v=\eta$ control results respectively. The parameters are $\alpha_{0}=1$ and $\beta_{0}=0 ; v=0.004$ for classical and Braginskii viscosity.

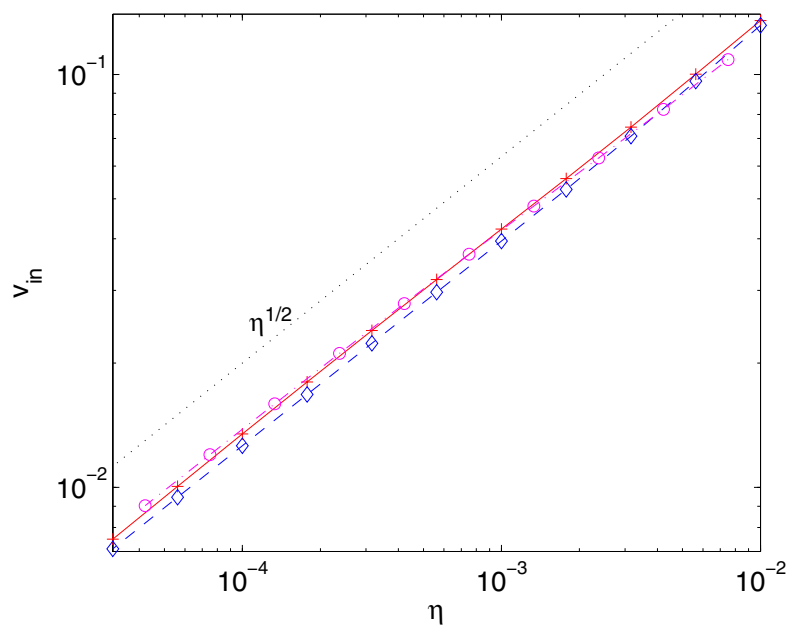

Fig. 5. Current sheet inflow speed comparisons. Crosses, diamonds and circles refer to Braginskii, classical and $v=\eta$ control results respectively. The parameters are $\alpha_{0}=1$ and $\beta_{0}=0 ; v=0.004$ for classical and Braginskii viscosity.

thickest current layer but the effect is marginal. This should be contrasted with the prediction $x_{s} \sim(\eta v)^{1 / 4}$ that follows from Sweet-Parker style arguments (Park et al. 1983).

The relation $x_{s} \sim \eta^{1 / 2}$ for the sheet thickness, coupled to the constraint $B_{s} \simeq 1$, leads to flux transfer and Ohmic dissipation rates that also follow the $\eta^{1 / 2}$ trend. These scalings are confirmed numerically for all three regimes (the $v=\eta$ control, the classical and Braginskii viscosities).

More interesting is the behaviour of the global viscous losses $W_{v}$. As indicated in Fig. 7, the Braginskii and classical viscous losses are effectively invariant, but the $v=\eta$ control strongly decreases with $\eta$. The decline of the $v=\eta$ control, however, is slower than the expected $W_{v} \sim \eta$ scaling. We have confirmed that this behaviour is due to strong vortical flows associated with the very weak levels of viscous damping. When we weaken the magnetic field, washed in by the flow, the distortion diminishes and the expected scaling is regained. Nevertheless, this behaviour

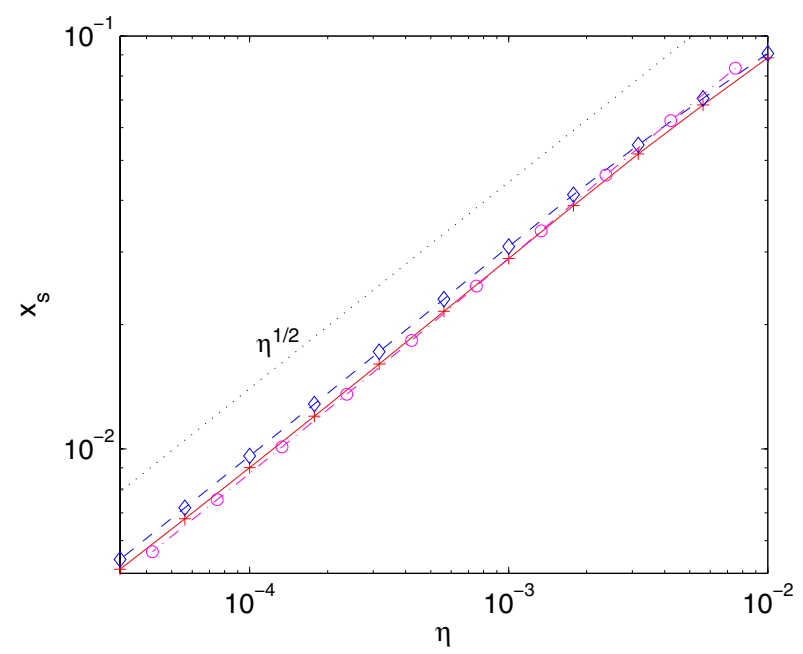

Fig. 6. Current sheet thickness comparisons. The dotted line shows $\eta^{1 / 2}$ scaling. Crosses, diamonds and circles refer to Braginskii, classical and $v=\eta$ control results respectively. The parameters are $\alpha_{0}=1$ and $\beta_{0}=$ $0 ; v=0.004$ for classical and Braginskii viscosity.

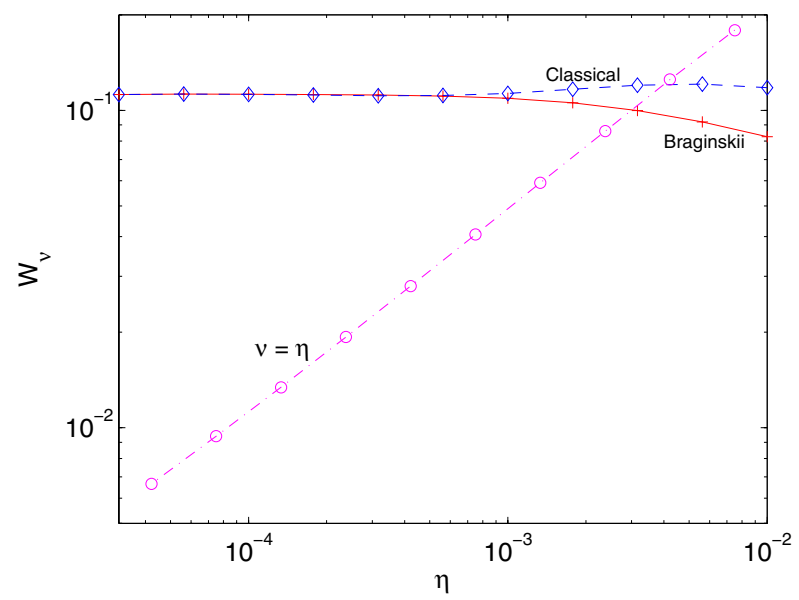

Fig. 7. Viscous dissipation rate comparisons. Crosses, diamonds and circles refer to Braginskii, classical and $v=\eta$ control results respectively. The parameters are $\alpha_{0}=1$ and $\beta_{0}=0 ; v=0.004$ for classical and Braginskii viscosity.

does indicate the enhanced sensitivity of the computation in the case of weak damping of the global velocity field.

Figure 8 plots the ratio of the viscous and resistive energy dissipation rates $W_{v} / W_{\eta}$. As anticipated by Eq. (18) in the case of fixed $v, W_{v} / W_{\eta}$ scales as $\eta^{1 / 2}$ as $\eta$ is reduced. We see that, independent of the form of the viscosity, $W_{v}$ exceeds $W_{\eta}$ by almost a factor of two at the lowest resistivity levels $\eta \leq 10^{-4}$.

In summary, all the resistive scalings presented in this section appear consistent with the simple analytical models of Sects. 3.1 and 3.2. That is, for the flow-driven reconnection simulations considered here, the resistive scaling laws are effectively identical for both the Braginskii and classical viscosities. The limited role of the traditional scaling arguments (Park et al. 1983) should be emphasised. Although they provide a reliable description of the reconnecting current sheet in a nonviscous plasma, they do not give a valid description of magnetic reconnection in a viscous plasma, at least in the present, flow-driven simulations. 


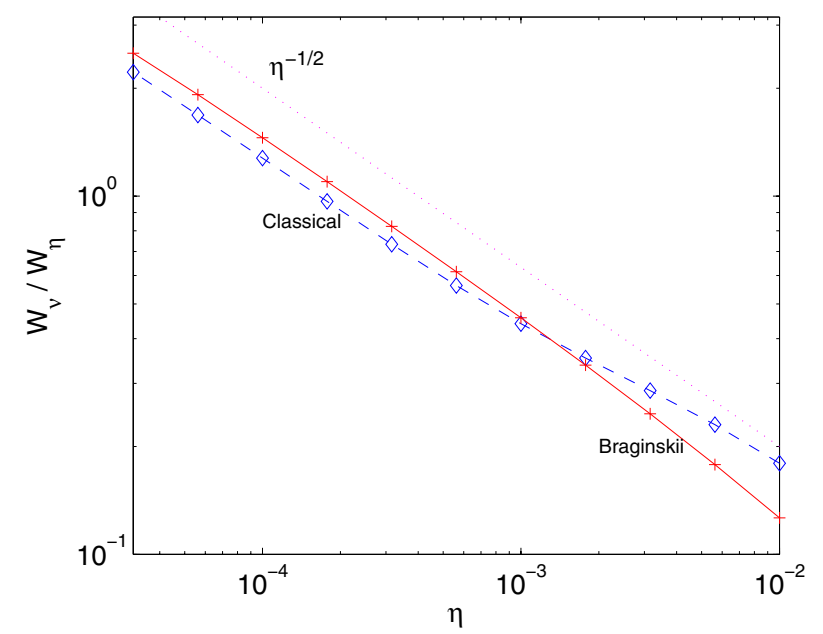

Fig. 8. The ratio $W_{v} / W_{\eta}$ for classical (diamonds) and Braginskii (crosses) viscosity. The dotted line shows $\eta^{-1 / 2}$ scaling. The parameters are $\alpha_{0}=1, \beta_{0}=0$ and $v=0.004$.

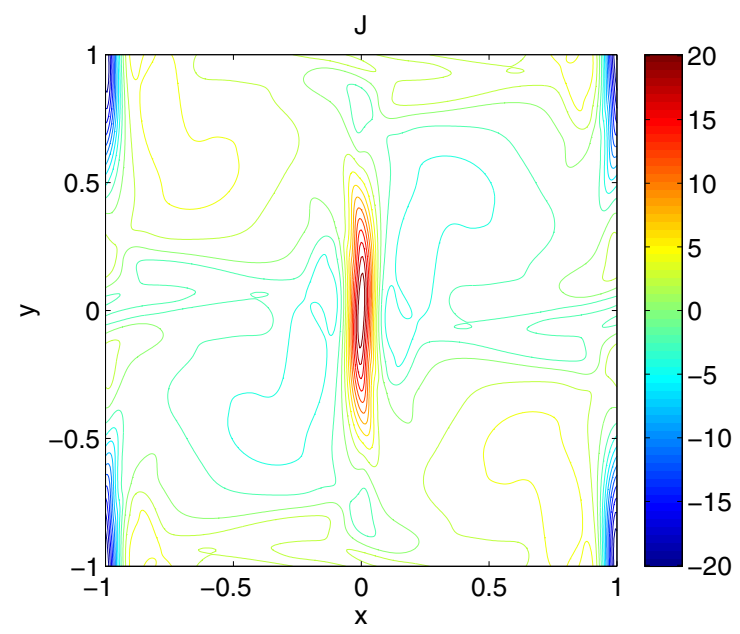

Fig. 9. Current contour plot for sheared reconnection in the Braginskii case at time of maximum current density at the origin. The parameters are $\alpha_{0}=1, \beta_{0}=0.7, \eta=0.004$, and $v=0.004$.

\subsection{Sheared magnetic reconnection simulations}

As pointed out previously, it is possible to construct an analytical model for sheared magnetic reconnection only in the nonviscous case (Craig \& Henton 1995). Guided by those exact resistive solutions, we expect that, compared with the head-on case, visco-resistive reconnection of sheared magnetic field lines should occur in thicker current sheets, which should lead to reduced energy dissipation and flux transfer rates. Figure 9 shows the current contour plot for a typical Braginskii run with a high value of shear $\left(\beta_{0}=0.7\right)$. As in the head-on case, there is a well defined current sheet in the vicinity of the magnetic null, but there is now significant warping of the ends of the sheet.

Our numerical results show that, even with relatively high levels of shearing, the classical viscosity results essentially follow the same scalings as in the head-on case $\left(x_{s} \sim \eta^{1 / 2}, W_{\eta} \sim\right.$ $\left.\eta^{1 / 2}, W_{v} \sim \eta^{0}\right)$. Systematic deviations, however, appear in the case of the Braginskii viscosity for high levels of shearing. These deviations from the analytical predictions imply that the structure of the current sheet is significantly modified by the anisotropic Braginskii viscous forces when the reconnecting

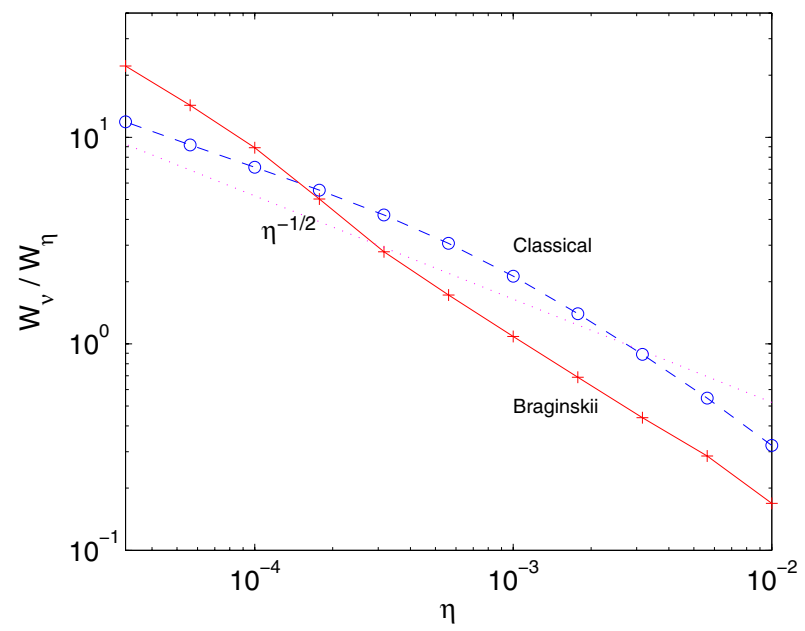

Fig. 10. The ratio $W_{v} / W_{\eta}$ for classical (diamonds) and Braginskii (crosses) viscosity. The dotted line shows $\eta^{-1 / 2}$ scaling. The parameters are $\alpha_{0}=1, \beta_{0}=0.7$ and $v=0.004$.

magnetic field lines are strongly sheared. Unfortunately, as yet, visco-resistive analytical solutions are only available for head-on reconnection.

Figure 10 illustrates the main new effect of strong magnetic shear (cf. Fig. 8). It shows $W_{v} / W_{\eta}$ for the classical and Braginskii viscosities in the case $\beta_{0}=0.7$. For the classical viscosity, both the resistive dissipation rate $W_{\eta}$ and the viscous dissipation rate $W_{v}$ agree with the analytical arguments, leading to Eq. (18). The ratio $W_{v} / W_{\eta}$ for the Braginskii viscosity, however, appears to follow a different scaling (closer to $\eta^{-1}$ ). We confirmed that this deviation is due to a slow down in the resistive dissipation rate (this scales closer to $\eta$ rather than $\eta^{1 / 2}$ ) in the strongly sheared Braginskii simulation.

\section{Conclusions}

Given the smallness of the electric resistivity in most astrophysical application, the search for a robust fast mechanism of magnetic reconnection has provided the motivation for a great deal of work on reconnection theory. Both analytical and numerical studies suggest that viscous dissipation may be capable of dominating resistive dissipation under a wide variety of coronal conditions (Hollweg 1986; Litvinenko 2005; Craig \& Litvinenko 2009). However, as we discussed in Sect. 3, analytical studies of magnetic reconnection in a resistive viscous plasma lead to contradictory results. Some analytical solutions predict the formation of a current layer on the visco-resistive scale $\sim(\eta v)^{1 / 4}$, while other solutions do not contain this scale. This key difference leads to different predictions for the parameters of the reconnecting current sheet and the dependence of the energy release rate on the viscous and resistive coefficients.

In this paper, we attempted to clarify the issue by performing numerical simulations of planar magnetic reconnection in periodic geometry. We considered head-on reconnection, driven by large-scale vortical flows in an incompressible plasma. We used both the classical shear viscosity and the Braginskii form for the ion parallel viscosity in a magnetised plasma. Somewhat surprisingly, our numerical results show that the parameters of the reconnecting current sheet (its thickness, the inflow and outflow speeds) are accurately described by purely resistive analytical scalings of flux pile-up magnetic merging, regardless of the form 
of the viscous stress tensor. The computed global resistive and viscous energy dissipation rates also closely follow Eq. (18), confirming that the current sheet thickness is effectively independent of viscosity for both the classical and Braginskii forms.

We have also made a preliminary study of the properties of the reconnection region in the case of a strong magnetic shear. It appears that in the Braginskii case magnetic shearing can modify the dissipation scalings, implying that the properties of the reconnecting current sheet depend on the form of the viscosity. This potentially important result requires further investigation. Interpreting numerical results in strongly sheared reconnection is not straightforward, since the available visco-resistive analytical solutions are only able to describe head-on merging.

To summarise, we find no evidence of the visco-resistive scale $\sim(\eta v)^{1 / 4}$ and the corresponding energy release rate scalings in our simulations of planar magnetic reconnection in periodic geometry. This finding contrasts sharply with predictions of some steady analytical models, Sweet-Parker style scaling arguments, and time-dependent simulations in a closed $X$-point geometry. There appears to be no simple criterion for the emergence of the visco-resistive scale.

Our results may be partly due to there not being enough time for the visco-resistive scale to develop: we took the current sheet parameters at the time of the first current maximum (first implosion), and it may be that the scale would develop after several implosions. Either way, the precise conditions required to ensure the emergence of a visco-resistive scale remain unclear. We believe that further analytic and numerical work is warranted, given the potential importance of viscous dissipation arising from either classical or Braginskii viscosity in solar flares and other astrophysical applications.

Acknowledgements. This work was supported by NSF grant ATM-0734032 and NASA grant NNX08AG44G.

\section{References}

Besser, B. P., Biernat, H. K., \& Rijnbeek, R.P. 1990, Planet. Space Sci., 38, 411 Biskamp, D. 1994, Phys. Rep., 237, 179

Braginskii, S. I. 1965, Rev. Plasma Phys., 1, 205

Craig, I. J. D. 2010, A\&A, 515, A96

Craig, I. J. D., \& Henton, S. M. 1995, ApJ, 450, 280

Craig, I. J. D., \& Litvinenko, Y. E. 2009, A\&A, 501, 755

Craig, I. J. D., \& Litvinenko, Y. E. 2010, ApJ, 725, 886

Craig, I. J. D., Litvinenko, Y. E., \& Senanayake, T. 2005, A\&A, 433, 1139

Heerikhuisen, J., Craig, I. J. D., \& Watson, P. G. 2000, Geophys. Astrophys. Fluid Dyn., 93, 115

Hollweg, J. V. 1985, J. Geophys. Res., 90, 7620

Hollweg, J. V. 1986, ApJ, 306, 730

Hosking, R. J., \& Marinoff, G. M. 1973, Plasma Phys., 15, 327

Jardine, M., Allen, H. R., Grundy, R. E., \& Priest, E. R. 1992, J. Geophys. Res., 97, 4199

Litvinenko, Y. E. 2005, Sol. Phys., 229, 203

Litvinenko, Y. E., \& Craig, I. J. D. 2000, ApJ, 544, 1101

Litvinenko, Y. E., \& Craig, I. J. D. 2003, Sol. Phys., 218, 173

Montgomery, D. 1992, J. Geophys. Res., 97, 4309

Park, W., Monticello, D. A., \& White, R. B. 1983, Phys. Fluids, 27, 137

Parker, E. N. 1957, J. Geophys. Res., 62, 509

Priest, E. R., \& Forbes, T. 2000, Magnetic reconnection: MHD theory and applications (Cambridge Univ. Press)

Rüdiger, G., \& Kitchatinov, L. L. 1997, Astron. Nach., 318, 273

Sonnerup, B. U. Ö., \& Priest, E. R. 1975, Plasma Phys., 14, 283

Titov, V. S., \& Priest, E. R. 1997, J. Fluid Mech., 348, 327 